\title{
Protée
}

\section{De l’iconicité aux scénarios iconiques}

\section{Les multiples chemins du " logos aisthêsis » dans l'œuvre d'Aristote}

\section{William Fiers}

Volume 30, numéro 2, automne 2002

Sémiologie et herméneutique du timbre-poste

URI : https://id.erudit.org/iderudit/006735ar

DOI : https://doi.org/10.7202/006735ar

Aller au sommaire du numéro

\section{Éditeur(s)}

Département des arts et lettres - Université du Québec à Chicoutimi

ISSN

0300-3523 (imprimé)

1708-2307 (numérique)

Découvrir la revue

Citer cet article

Fiers, W. (2002). De l'iconicité aux scénarios iconiques : les multiples chemins du « logos aisthêsis » dans l'œuvre d'Aristote. Protée, 30(2), 95-110.

https://doi.org/10.7202/006735ar

\section{Résumé de l'article}

Cet article propose une réinterprétation, dans le cadre de la sémiotique " tensive ", de la notion d' " iconicité " à partir d'une analyse approfondie des réseaux iconiques, tels qu'ils sont décrits par Aristote dans De Anima et « De Sensu ». Loin d'être le résultat d'une homologation arbitraire à travers une grille de lecture conceptuelle (Greimas) ou d'un codage sous forme de stimuli substituts (Eco), nous allons montrer que l'effet de l'iconicité est le résultat d'une finalisation esthétique d'un processus sensoriel s'instaurant depuis le maintien en présence d'une perception constitutive. Cette première stabilisation du flux sensoriel ouvre une perspective égocentrique susceptible de procurer une cohérence à la sensorialité par l'intermédiaire de ce qu'Aristote appelle la koinè aisthêsis. À partir des textes d'Aristote, nous allons faire l'hypothèse que cette cohérence s'obtient de quatre façons différentes.
Ce document est protégé par la loi sur le droit d'auteur. L'utilisation des services d’Érudit (y compris la reproduction) est assujettie à sa politique d'utilisation que vous pouvez consulter en ligne.

https://apropos.erudit.org/fr/usagers/politique-dutilisation/ 


\section{DE L'ICONICITÉ AUX SCÉNARIOS ICONIQUES LES MULTIPLES CHEMINS DU «LOGOS AISTHÊSIS» DANS L'CEUVRE D'ARISTOTE}

WILLIAM FIERS

La problématique de l'iconicité et de l'iconisation est l'une des plus épineuses et, en même temps, l'une des plus débattues dans la théorie de la sémiotique. Elle a amené, à la fin des années 1960, à une véritable controverse méthodologique portant notamment sur le caractère analogique de l'icône. Nous allons tout d'abord retracer brièvement les grandes lignes du débat afin de mieux situer notre propre contribution.

Constatons d'emblée que la controverse est due au fait que, depuis toujours, la sémiotique d'inspiration peircienne revendique l'iconicité comme son domaine d'expertise et qu'elle considère l'iconicité dès lors comme un signe faisant partie de la triade "indice-icône-symbole» ${ }^{1}$. Tandis que l'indice est un signe d'existence (la fumée indiquant le feu), l'icône n'indique que la possibilité de l'existence d'une chose à travers un certain degré d'isomorphisme. La chose se maintient en présence par l'intermédiaire de l'icône comme un schème spatio-temporel, qui reproduit la perception sous la forme d'une image analogique. Le symbole réduit finalement l'effet de vérité au statut du pensable, aux règles et aux codes ne permettant pas plus qu'une possibilité de reconnaissance ${ }^{2}$.

S'ajoutent la domination du paradigme linguistique et ses modèles, qui ont fait office de critère d'évaluation pour le fonctionnement sémiotique de tous les systèmes de signes, verbaux et non verbaux, ce qui a eu pour conséquence une lexicalisation de la perception amenant à une césure entre le domaine propre à l'image et le domaine propre à la langue. Le résultat étant une perte de la compréhension du fonctionnement spécifique des codes analogiques de l'image, car la lexicalisation de la perception à l'aide des modèles linguistiques passe totalement outre à la capacité des effets sensoriels de l'image à produire sui generis des effets iconiques et "n'analyse que le discours dont telle image ou tel tableau est l'objet, non les données plastiques qui les définissent dans leur spécificité» ${ }^{3}$. Comme les premiers sémiologues considèrent tout ce qui relève dans l'image d'une analogie comme non codé et, en conséquence, non pertinent pour la sémiosis, et tout ce qui relève de l'arbitraire comme codé, alors pertinent pour la sémiosis, les éléments analogiques ne peuvent produire une signification qu'à travers un processus de codage. Celui-ci représente, selon Christian Metz, la condition sine qua non de la sémiosis:

c'est au-delà de l'analogie que le travail du sémiologue peut commencer, faute de quoi on pourrait craindre [...] qu'il n'y ait plus rien à dire de l'image, sinon qu'elle est ressemblante. ${ }^{4}$

Au début des années 1970, lorsqu'Umberto Eco soumet le concept d'iconicité à une révision, le débat entre dans une nouvelle phase. Selon lui, il n'y a aucune raison d'admettre que le signe iconique soit motivé, c'est-à-dire qu'il possède des caractéristiques analogiques susceptibles de reproduire des objets du monde naturel. Le signe iconique reproduit, comme le dit Eco, tout au plus

[...] quelques conditions de la perception commune, sur la base des codes perceptifs normaux et en sélectionnant ces stimuli qui - d'autres stimuli étant éliminés - peuvent permettre de construire une structure perceptive qui possède- 
par rapport aux codes de l'expérience acquise - la même signification que l'expérience réelle dénotée par le signe iconique. ${ }^{5}$

En d'autres termes, les stimuli sensoriels des signes iconiques, codant un objet dans le monde réel, n'ont pas tant trait à ce qui est directement perceptible, mais sollicitent plutôt les schèmes mentaux permettant la reconnaissance d'un objet et sa valeur sémiotique. Le signe iconique reproduit dès lors seulement ces traits de l'objet réel qui sont homologues «au modèle de relations perceptives que nous construisons en connaissant et en nous rappelant l'objet " ${ }^{6}$. Vingt ans après cette publication, Eco ne juge pas nécessaire de revoir ses hypothèses. En envisageant la problématique de l'iconicité dans le cadre de «l'hypoiconicité» de Peirce, c'est-à-dire comme une procédure sémiotique quasi universelle déterminant le rapport d'homomorphie entre représentation et représenté, il la rapproche "d'une théorie établie de la similarité, c'est-à-dire [de] celle de la création de l'effet de ressemblance» à travers des "stimuli substituts» susceptibles de reproduire «quelques-unes des conditions de la perception de l'objet [et de mettre] en jeu les mêmes inférences perceptives que celles mises en jeu pour percevoir l'objet réel» 7 .

Le processus de codage est considéré par A.J. Greimas, à son tour, comme une transformation d'un niveau sémiotique non linguistique - la perception - en un niveau sémiotique linguistique. Greimas postule que la catégorisation de la perception du monde réel est conforme à celle de divers codes sensoriels (picturaux, filmiques, linguistiques) dans la mesure où l'une comme l'autre s'obtiennent à travers les mêmes figures sensorielles. La façon dont nous observons les objets et les signifions dépend des figures que la forme des diverses sémiotiques met en place:

[...] la transformation de l'expression en contenu, considérée comme une procédure de la mise en corrélation de deux systèmes virtuels - dont l'un commande le procès de la perception et dont l'autre rend compte de la manifestation linguistique de la structure sémantique -, peut être présentée comme une tentative d'explication du passage du référent extra-linguistique au plan du contenu linguistique, c'est-àdire à la structure sémantique. 8

Pour Greimas, le processus de l'iconisation du perceptible s'inscrit dans la problématique de la sémiotique du monde naturel. En remplaçant le monde naturel contingent des substances par un niveau morphologique, la sémiotique greimassienne considère ce monde comme une sémiotique à part entière, ayant une forme de l'expression et une forme du contenu, dont les figures sensorielles agissent sur l'homme sans aucune intervention linguistique. Ces figures ne sont pas des signes, mais des unités formelles qui font partie d'un réseau relationnel; elles forment le soubassement catégoriel sur lequel s'ancre la grille de lecture que le percevant projette sur le monde afin de lui donner une signification. «De nature sémantique - et non visuelle, auditive ou olfactive par exemple -, elle sert de "code" de reconnaissance qui rend le monde intelligible et maniable»9. Si le monde phénoménal est compris à travers la perception comme une morphologie de qualités sensorielles, la projection d'une grille de lecture - sorte de «signifié du monde», comme le dit Greimas - actualise l'existence virtuelle d'une structure sémantique et permet la reconnaissance thématique iconique du figuratif. En l'occurrence, l'iconicité est à considérer comme un principe d'homologation entre le plan du contenu et le plan de l'expression et non comme la mise en discours de figures sensorielles toutes faites, directement issues du monde naturel.

Or, qu'il s'agisse d'une analyse de l'iconicité de la sémiotique du monde naturel ou d'une sémiotique construite (littéraire, picturale, cinématographique), la lexicalisation des formants figuratifs en objets de sens entraîne une perte de la spécificité propre au niveau figuratif, dans la mesure où cette spécificité coïncide avec son identification lexicale sans que celle-ci épuise la totalité des articulations signifiantes de la perception.

Dans les années 1980, les carences de la sémiotique figurative ont été mises à nu, notamment par Jean-Marie Floch. Selon lui, le signifiant visuel permet avant tout une lecture proprement plastique des images, c'est-à-dire des phénomènes visuels analogiques qu'une lecture purement figurative virtualise afin d'établir une lexicalisation et de produire la sémiosis. La sémiotique plastique, que propose Floch, se constitue ainsi

[...] sur le constat de l'insuffisance d'une seule approche "figurative " à rendre compte de la signification des tableaux, photographies ou même de certains plans d'architectures transformés en objets "esthétiques». ${ }^{10}$

En effet, s'il s'agit de déterminer la signification des images figuratives abstraites, la lecture figurative bute, selon Floch, 
[...] sur des contradictions visuelles qu'actualise une lecture plastique. Tout se passe [...] comme si la lecture figurative s'avérait incapable de relier, d'enchainer les signes qu'elle a constitués en tant que sémiosis, et ce du fait de la résistance ou du détournement du signifiant de certains d'entre eux. ${ }^{11}$

Contrairement à la sémiotique figurative, la sémiotique plastique ne part pas du postulat d'une existence d'une grille de lecture conceptuelle antérieure à la description du signifiant, mais de l'idée que le signifiant est avant tout un ensemble d'unités plastiques dont le signifié s'obtient $a$ posteriori, c'est-à-dire à travers l'isomorphisme semisymbolique s'établissant à partir de la forme de l'expression, sans que celle-ci soit nécessairement lexicalisée comme une langue naturelle.

La sémiotique plastique [...] est issue d'une volonté de rendre compte de la matérialité du signifiant des images et des espaces bâtis et, plus généralement, d'une interrogation sur les modes d'existence sémiotique des "logiques du sensible", pour reprendre l'expression de Claude Lévi-Strauss. 12

En bref, la sémiotique plastique s'interroge sur la manière dont le niveau de l'expression peut générer une signification, c'est-à-dire comment on peut passer d'une sensation à une perception, puis à une interprétation indépendamment $\mathrm{du}$ plan du contenu attaché à l'expression par convention.

Le retour à la sensation, comme point de départ d'un processus d'iconisation obéissant à ses propres lois, marque l'avènement de la sémiotique tensive. Le fait que les phénomènes sensoriels de la manifestation relèvent d'une tensivité constitutive, dont l'instance est le sujet sentant, a déjà été constaté par Greimas et Fontanille dans Sémiotique des passions ${ }^{13}$. Mais, dans celle-ci, la corporéité est encore liée à l'intelligibilité de l'expérience. Le corps sentant n'est qu'une simple instance médiatrice entre l'expérience et sa lexicalisation et se résume à un simple regard ou point de vue assumant la discrétisation et la catégorisation de l'expérience.

En mettant le corps au centre de la réflexion ${ }^{14}$, la sémiotique s'ouvre finalement à une approche qui prend en considération l'effet de la présence corporelle sur la sémiosis ellemême. Celle-ci ne sera plus, dès lors, une fonction réglée par la présupposition logique du schéma narratif visant l'intelligibilité par l'isomorphisme, mais une fonction réglée sur le corps et ses structures sensori-motrices. Il s'ensuit que l'iconicité ne peut être un effet de ressemblance arbitraire s'établissant sur la base d'une homologation opérée par la grille de lecture, elle représente plutôt le résultat d'une corrélation qui s'obtient sur la base de la sensori-motricité stabilisant momentanément une zone de conflit entre la globalité du flux sensoriel et ses seuils locaux ${ }^{15}$.

\section{Le «logos aisthêsis» d'Aristote}

Dans la deuxième partie de notre travail, nous proposons de mettre au clair les multiples façons dont cette corrélation sensori-motrice stabilise, puis finalise le flux sensoriel de manière à établir des scénarios iconiques ayant leur propre logique. Cette logique de la sensation, nous allons la chercher chez Aristote. Dès lors qu'Aristote constate que le "blanc» est «ici», qu'il est "grand» et qu' il s'agit du "fils de Diarès», ou que "amer» et "jaune» font du "fiel», on peut dire qu'il traite la sensation comme un objet de connaissance sémiotique. L'analyse des moments iconiques propre à la sémiotisation de la sensation a comme point de départ le maintien en présence d'un stimulus sensoriel. Celui-ci ouvre une perspective égocentrique susceptible de procurer une cohérence au monde sensoriel qui va bien au-delà de ce qui est directement sensible et qui présuppose alors, non seulement un pouvoir critique discriminatoire, mais également l'existence de sensibles communs, à savoir des qualités intermodales perceptibles par plusieurs sens, voire une capacité de pondération transmodale, qui totalise l'expérience sensorielle et qui permet d'établir des objets de sens à caractère synesthésique. Nous allons voir que ce pouvoir critique et la capacité de pondération sont inhérents, selon Aristote, à la logique même de la sensation, et qu'ils déploient quatre opérations sémiotiques dynamiques - la discrétisation, l'intégration, la concentration et l'unification - qui prennent en charge l'articulation axiologique du champ sensoriel. Ces opérations relèvent d'autant de modulations neuro-sensorielles sous-jacentes, qui émergent à partir de l'activité sensori-motrice et perceptive du percevant à l'égard d'une seule réalité sensorielle.

En suivant les analyses d'Aristote présentées dans $D e$ Anima et "De Sensu", nous allons faire l'hypothèse qu'il y a également quatre types de connexions qui sous-tendent l'établissement de cette axiologie, à travers lesquelles la sensation «iconise» et acquiert un statut autonome vis-àvis de sa substance, à savoir les organes et les stimuli sensoriels. 
L'empreinte monodimensionnelle de la sensation

Pour Aristote, l'Être existe à travers le sensible: il est présent de diverses façons dans l'individu et dans la nature. Le sensible est le point de départ d'un chemin de la connaissance. À partir de ce qui est clair "pour nous", c'est-àdire immédiatement connu et seulement esthétiquement marqué, il nous fait accéder à ce qui est clair «en soi», c'està-dire ontologiquement marqué. Le sensible, pour Aristote, est le premier connu représentant l'universalité de l'expérience où tout est mélangé; l'analyse a pour tâche de le déterminer en ses particularités distinctives et intelligibles ${ }^{16}$.

Ce qui est crucial dans la pensée d'Aristote, c'est que la raison humaine peut saisir, par l'intermédiaire des sens, l'essence d'une chose à travers sa forme sensorielle.

D'une façon générale pour toute sensation, il faut comprendre que le sens est le réceptacle des formes sensibles sans la matière, comme la cire reçoit l'empreinte de l'anneau sans le fer ni l'or, et reçoit le sceau d'or ou d'airain, mais non en tant qu'or ou airain; il en est de même pour le sens: pour chaque sensible, ilpâtit sous l'action de ce qui possède couleur, saveur ou son, non pas en tant que chacun de ces objets est dit être une chose particulière, mais en tant qu'il est de telle qualité et en vertu de sa forme. ${ }^{17}$

Selon Aristote, la présence de la forme sensible dans l'objet laisse son empreinte sur l'organe du percevant. La perception physique de la forme sensible consiste alors en la présentification, dans l'organe du sens, du logos qui caractérise la forme sensible. Chaque sens est spécialement destiné à recevoir tel genre de sensibles à l'exclusion des autres. En l'absence d'un sens particulier, ce sens ne peut être reconstruit par voie de transmodalité: un sens, comme le veut Aristote, est irrémédiablement monodimensionnel.

Cette monodimensionnalité de la sensation implique qu'il y a une symétrie parfaite entre les stimuli du monde et les réceptacles sensoriels du percevant, opérée par ce qu'Aristote appelle la sensation commune. Celle-ci n'est qu'une simple synthèse de sensations suscitées par un même objet offert aux divers sens: cet objet s'entend et se palpe en même temps, sans qu'il y ait plus qu'une association des différentes sensations ${ }^{18}$. Selon Aristote, comme il s'agit d'une expérience dérivée d'une seule essence, on peut transposer, grâce à la sensation commune, une qualité sensible d'un registre sensoriel à l'autre. Par contre, il ne peut y avoir une complexité intermodale à l'intérieur de la sensation que nous procure un seul canal sensoriel, car il s'agit toujours d'une expérience littérale - on sent le son aigu qui nous coupe littéralement - qui s'obtient à partir d'une seule essence.

\section{«Hylê-morphisme» et le mouvant-mû}

La pensée d'Aristote est ce qu'on appelle hylê-morphologique. La forme est solidaire de sa substance. La forme est la substance en acte, qui, à son tour, incorpore cette forme déjà en puissance. La forme et la substance sont donc identiques en puissance et multiples en acte. D'où l'intuition fondatrice d'Aristote, qui veut que ce qui est numériquement un (en réalité) est logiquement multiple (en pensée). Dès lors, la substance est un composé susceptible d'être pensé en tant que substance ou matière première et en tant que forme ou materia signata. En tant que puissance, le composé de tous les objets, naturels et animés, incorpore, par essence, à la fois le principe et la cause d'un mouvement à travers lequel se produit sa forme sensorielle actuelle aussi bien que toutes les formes futures virtualisées par cette actualisation. Le composé est susceptible et cause d'un changement sui generis, en tant qu'il se meut et se trouve mû, ce qui a pour conséquence qu'il est pensé sous un double aspect: en tant qu'il se meut et renferme la cause de son mouvement, et en tant qu'il est mû et subit le mouvement dont il est la cause ${ }^{19}$.

Le hylê-morphisme a pour but d'expliquer pourquoi la substance numériquement «un» est justement devenue telle forme spécifique, autrement dit, pourquoi il y a changement. Comme le constate Aristote:

Il faut que le même être soit en un sens immobile et en un sens mû. Le mû est mû grâce à son point d'appui, comme si c'était une des parties qui était mise en mouvement. Car une partie s'appuie sur l'autre comme sur un point fixe. ${ }^{20}$

Cependant, on ne saurait attribuer à ce point fixe une partie déterminée du corps naturel. Il s'agit plutôt d'une fonction déplaçable, dont le siège est à la fois l'âme et ce dans quoi elle réside, à savoir le corps. En conséquence, la présentification d'une sensation n'est pas à considérer comme le résultat d'un processus unilatéral. La saisie de l'empreinte est une activation dans l'organe sensitif de la forme sensible appartenant à la substance d'une chose, qui, à son tour, est susceptible d'être activée ou mue par l'organe sensitif afin de "rendre" sa forme. La saisie de la forme, c'est-à-dire son devenir présent à travers ses diverses esquisses, et sa visée, c'est-à-dire sa reconstruction iconique, 
produisent une seule existence réelle. L'objet sensible et le réceptacle sensoriel sont substantiellement différents l'un de l'autre, mais ont toujours la même réalité formelle en commun; un seul état qualitatif exprimant l'acte (la forme) de ce qui est en puissance (le composé substance-forme). Autrement dit, l'actualisation d'une sensation est le résultat d'une mise en commun sous une seule forme sensible de deux composés, qui, l'un comme l'autre, d'une façon réversible, agissent et pâtissent, meuvent et sont mus.

\section{L'«aisthêsis»}

Selon Aristote, dans l'expérience sensorielle, l'objectif et le subjectif sont réciproques: l'aisthêsis dit à la fois la faculté de percevoir (la sensibilité), l'exercice de cette faculté (la perception), sa distribution liée et non liée aux organes des sens (les cinq sens canoniques) et les affections ponctuelles, les pathèmes (les sensations), produits par les objets de sens. L'aisthêsis est une coöncidence entre l'activation de l'organe du sens (aisthêtêrion) et l'objet sensible (aisthêton), il affirme l'identité entre sensibilité et sensible: «l'acte du sensible et celui du sens sont un seul et même acte, mais leur quiddité n'est pas la même» ${ }^{21}$. Cette identité formelle $s^{\prime}$ instaure à travers le mouvement de la sensation. Non identiques en essence de par leurs substances matérielles différentes, la faculté sensitive et le senti deviennent identiques en acte. L'aisthêsis opère dès lors une dématérialisation de la substance primaire pour en faire un «senti» (logos: une forme intelligible) susceptible d'être soumis à une pondération discriminatoire.

En conséquence, l'aisthêsis est surtout un "pouvoir critique», qui intervient dès qu'il est question de sensation; elle juge en effectuant un calcul du rapport entre les contraires qui caractérisent un senti propre en acte. Autrement dit, elle fait la moyenne de ce qu'elle sent; par exemple, «trois fois plus de noir que de blanc fait du gris» 22 . En tant que telle, la logique de la sensation est un calcul arithmétique, qui s'opère à travers les différences que l'organe sensoriel retient des sentis.

\section{Les sensibles selon la logique de la sensation}

\section{Définitions:}

Le sensible désigne trois espèces d'objets: deux de ces espèces sont, disons-nous, perceptibles par soi, tandis que la troisième l'est par accident. ${ }^{23}$

En outre, des deux espèces de sensibles par soi, ce sont les sensibles propres qui sont des sensibles proprement dits, et c'est à eux qu'est adaptée naturellement la substance de chaque sens. ${ }^{24}$ C'est par accident, en effet, que l'on perçoit [le fils de Diarès], parce qu'au blanc est accidentellement uni l'objet senti. 25 Car il n'appartient certainement pas à un autre sens [que la sensation commune] de prononcer que ces deux qualités ne font qu'une seule chose. ${ }^{26}$

L'expérience sensible s'obtient par l'intermédiaire de trois sortes de sensibles et par ce qu'Aristote appelle la «sensation commune», qui assure leur unité27. À l'instar d'Aristote, nous distinguons quatre types sensoriels dont nous allons dégager le logos pour présenter une synthèse susceptible de répondre à la question suivante: comment la signification vient-elle à la sensation?

Ces quatre types sont, respectivement, le sensible propre, le sensible commun, le sensible par accident et la sensation commune.

\section{Le sensible propre}

Le sensible propre à chaque sens est un perceptible par soi, en ce qu'il représente une qualité qui tombe directement sous le sens. Le processus de captation, grâce auquel la faculté sensorielle s'actualise comme champ sensoriel, instaure un processus de privation, une séparation constitutive entre les formes et la matière du composé. Dans le même temps la privation engendre un potentiel, le champ sensoriel proprement dit, dont la forme sensible actualisée virtualise toutes les futures esquisses.

Aristote qualifie ce processus d'activation comme un "pâtir» de l'organe sensoriel qui subit l'empreinte du composé dans l'acte de la sensation; mais ce "pâtir» incorpore une activité de progrès vers un état "positif»: l'organe sensoriel non seulement reçoit la forme de l'empreinte, mais la provoque, la conserve et, de cette manière, «apprend» et juge la sensation qu'il retient du composé28.

Ce jugement n'est pas un jugement d'erreur ou de vérité, mais un jugement d'existence: le blanc que capte l'œil est une constatation, qui est en soi ni vraie ni fausse, mais tout simplement, épistémologiquement parlant, neutre, dans la mesure où sa perception est indépendante des ressources conceptuelles et des mécanismes de formation de croyances d'un individu.

Ainsi, la sensation peut être conçue comme l'existence formelle d'un substrat matériel, qui est le corps ${ }^{29}$. La capa- 
cité d'un corps à être le substrat d'une sensation, à savoir les facultés sensorielles, se présente à son tour comme une structure également formelle associée à la forme sensorielle, que le percevant retient du substrat. L'unité formée par le substrat matériel du corps et ses facultés sensorielles constitue une forme-résidu à caractère organisateur: l'âme, "ce par quoi nous vivons, percevons et pensons [et qui] sera notion et forme, et non pas matière et substrat " ${ }^{30}$. La sensation est une fonction commune à l'âme et au corps. Elle n'appartient donc ni à l'un ni à l'autre, "car ce dont il y a puissance est aussi ce dont il y a acte, et ce qu'on appelle sensation, en tant qu'acte, est un certain mouvement de l'âme par l'intermédiaire du corps » ${ }^{31}$.

Son existence s'affirme alors en tant que principe vis-àvis des organes sensitifs et en tant que champ sensoriel formel vis-à-vis du monde sensible. Ce champ sensoriel n'est pas homogène, mais d'emblée régi par deux dimensions qui l'orientent selon le point de vue que l'on adopte:

1. Selon le point de vue de la substance, il s'agit du devenir vers la réalisation de la sensibilité à travers les diverses strates des modes d'existence. Un mouvement global que l'on pourrait identifier à un devenir "autre», indiquant un changement de ce qui demeure numériquement «un». L'essence du substrat, ce qu'Aristote identifie à l'âme, ne cesse de devenir "un" à travers les formes qu'elle retient de la substance matérielle.

2. Le devenir continuel, qui caractérise cette invariance du substrat, se recoupe avec un deuxième devenir: celui de la variance, qui fait apparaître la diversité des attributions successives du substrat et qui porte sur un mouvement local à travers des couches, des existences morphologiques, ayant pour résultat une alternance qualitative répétitive entre deux formes. Un devenir que l'on pourrait identifier, à l'instar d'Aristote, au devenir «multiple» du substrat.

On constate que le devenir «un» et le devenir «multiple» instaurent deux sortes de mouvements qui contrôlent le champ sensoriel propre au sensible propre:

a) Un contrôle non linéaire qui garantit la stabilité de la manifestation perceptive et, par conséquent, un degré permanent d'acuité globale du champ sensoriel, en «reparamétrant» les formes sensorielles que celui-ci retient de la substance matérielle.

b) Un contrôle linéaire qui relève d'un mouvement métrique spatio-temporel local, qui est solidaire des divers états sensoriels qui surviennent dans le champ sensoriel.
On a affaire alors à une convergence entre, d'une part, un mouvement linéaire physique à travers les couches proprement sensorielles et, d'autre part, à un mouvement téléologique à travers les strates des modes d'existence qui paradigmatisent le champ de la sensation. Celui-ci est d'abord un espace tensif, dans la mesure où il est le résultat d'une interaction polysensorielle au niveau local de la sensation. Ainsi, l'actualisation continuelle des impressions sensorielles contraires garantit l'émergence et le maintien en permanence d'un espace qualitatif global où toutes les esquisses futures, dans lesquelles le champ sensoriel peut se réaliser, sont virtualisées.

C'est l'ensemble de la sensation, c'est-à-dire la pluralité de ses incidences sensorielles, qu'Aristote identifie à l'actualisation d'une unité potentielle, celle de l'âme. Pour lui, la sensation n'est pas un pur phénomène physique, mais avant tout une affection de l'âme. Ce n'est pas l'œil en tant que tel qui voit, mais l'âme, par l'œill ${ }^{32}$. L'âme représente l'invariance du champ sensoriel du percevant - son essence - à travers la variance morphologique que celui-ci retient du substrat matériel. La sensation, à son tour, en tant qu'elle caractérise l'âme, est le premier stimulus d'une chaîne de réactions internes qui amène à une finalisation iconique du sensible. Mais elle représente pour l'homme bien plus qu'un simple mode de connaissance; elle constitue son activité caractéristique, sa manière de vivre et sa forme de vie.

\section{Modulation}

L'actualisation d'un sensible n'est pas une simple suite de reproductions, mais bien une suite de représentations qui, au lieu d'être des exemplaires typiques d'un genre sensoriel, affirment à chaque fois leur propre logique et, $a$ fortiori, leur propre raison d'être. En effet, l'actualisation du jaune ne vaut pas l'actualisation du bleu, qui, à son tour, ne vaut ni l'actualisation d'un deuxième bleu ni celle d'un deuxième jaune ${ }^{33}$. Selon Aristote, l'aisthêsis incorpore un "pouvoir critique» opérant un calcul arithmétique ponctuel, qui évalue la proportion respective des contraires qui caractérisent tel sensible.

Le sensible propre s'obtient à travers un processus «sensori-cognitif" qui prend la forme d'un alliage ou d'un mélange ${ }^{34}$. Le devenir semblable de l'organe sensoriel "patient" et de l'objet sensible «agent", à travers la sensation, ne produit pas une composition, juxtaposition ou superposition, mais une «unification des choses mélangeables, à la suite 
de leur altération" 35 . L'équilibre qui s'établit virtualise toutes les qualités sensibles d'un même genre. Le sensible en acte se présente comme un mélange, une actualisation spécifique d'un complexe sensoriel, que l'on éprouve comme une seule qualité sensorielle homogène.

\section{Les médiums: le diaphane et la chair}

et les premières articulations du «logos aisthêsis»

Le passage d'un état virtuel à un état réalisé s'opère à travers ce qu'Aristote appelle un médium: diaphane, lorsqu'il s'agit de sensibles à distance (visibles, auditifs, olfactifs, à savoir les parfums), et chair, lorsqu'il s'agit de sensibles immédiats (tactiles, gustatifs, olfactifs, à savoir les aromates) ${ }^{36}$. Ces médiums actualisent ce qui est en puissance, à savoir les facultés sensorielles.

La vue, l'ouïe et les parfums peuvent être considérés comme des sens extéroceptifs qui spatialisent l'expérience sensorielle, dans la mesure où ils procurent au percevant les moyens de mesurer la distance entre lui et le monde et de déterminer la provenance des présences sensibles qui surgissent dans son champ sensoriel. En revanche, le goût, le toucher et les aromates sont considérés comme des sens proprioceptifs qui temporalisent l'expérience sensorielle, dans la mesure où ils sollicitent le corps, par l'intermédiaire de la chair, en tant qu'unité temporelle. Pour Aristote, il y a une différence profonde entre ce que l'on perçoit à une certaine distance du corps et ce que l'on perçoit sur le corps:

$[\ldots]$ pour les tangibles [...] la perception ne s'effectue pas sous l'action de l'intermédiaire, mais en même temps que l'intermédiaire, à la façon de l'homme frappé à travers son bouclier: ce n'est pas que le bouclier, une fois le coup reçu, ait frappé l'homme à son tour, mais, en fait, les deux coups se sont trouvés portés simultanément. ${ }^{37}$

Nous allons voir qu'à ces cinq sens viennent s'ajouter les sensibles communs (le mouvement sensori-moteur kinesthésique) et la sensation commune (ou conesthésique), aptes à intégrer les diverses sensations (extéroceptives et proprioceptives) et à nous faire sentir intéroceptivement ce que nous percevons à travers la sensation.

\section{Le diaphane}

Pour Aristote, toute sensation ne s'accomplit pas sans un médium ou un milieu, qui fonctionne en quelque sorte comme un filtre à travers lequel la forme sensible de l'objet senti est retenue. En ce qui concerne les «sensibles à distance", ce filtre est une qualité actualisée d'un corps transparent, ce qu'Aristote appelle un diaphane: un composé de matière et de forme, grâce auquel le filtre fonctionne effectivement comme un filtre.

Par diaphane, j'entends ce qui, bien que visible, n'est pas visible par soi, à proprement parler, mais à l'aide d'une couleur étrangère: tels sont l'air, l'eau et un grand nombre de corps solides [le verre]. Car ce n'est ni en tant qu'eau, ni en tant qu'air qu'ils sont diaphanes, mais parce que, dans l'un comme dans l'autre élément, se trouve une même nature. 38

Remarquons d'abord que pour Aristote le diaphane n'est pas en lui-même coloré, mais qu'il emprunte la couleur aux couleurs qu'il actualise. Il s'ensuit que la nature ontologique du diaphane est celle d'une matière sans corps et sans forme, c'est-à-dire d'une matière "sans lieu», car il n'existe pas d'étendue dans l'air, dans l'eau ou dans les corps solides susceptibles de l'abriter matériellement. Son existence est, par conséquent, purement conceptuelle; en bref, on a affaire à un composé intelligible 39 .

Précisons d'avantage la nature du diaphane. «L'acte du diaphane en tant que diaphane est la lumière» ${ }^{40}$, dit Aristote pour indiquer que la lumière est l'actualisation de la substance intelligible du diaphane. La lumière est l'acte du diaphane et la couleur n'est que ce qui est visible dans la lumière. Elle est "comme une couleur du diaphane», mais sans être la couleur elle-même de l'objet; en tant que diaphane en acte, la lumière est la condition sine qua non de la couleur: c'est la lumière qui rend saisissable ce qui est intelligible dans la couleur. En d'autres mots, une sensation engendrée par un diaphane s'accompagne toujours d'une intelligibilité ${ }^{41}$. Les sensibles à distance sont, pour ainsi dire, littéralement rendus extéroceptifs par le diaphane en acte.

\section{La chair}

En ce qui concerne le fonctionnement du médium chair, Aristote constate que celui-ci «semble se comporter à la façon d'une enveloppe d'air qui adhérerait naturellement à nous» ${ }^{42}$ et qu'elle est «l'intermédiaire de la faculté du toucher [du toucher en tant que tel et également du goût] à travers lequel se produit la multiplicité des sensations [tactiles]» ${ }^{43}$. 
Même dans le cas du toucher, il n'y a jamais contact direct entre la faculté sensitive et l'organe sensoriel. Dès lors que la chair n'est qu'un médium, une membrane, qui est à l'organe tactile (pour Aristote, le cœur) «ce que l'air et l'eau sont aux organes de la vue, de l'ouïe et de l'odorat " 44 : "quelle est la chose unique qui serait substrat du toucher, comme le son est substrat de l'ouie?» 45 .

Cette substance sous-jacente ne peut être autre chose que la tactilité dans sa globalité. C'est dire que la chair, en tant que médium qui nous est corporellement conjoint, engendre un champ sensoriel tactile dont la quiddité est semblable à celle du médium: «le toucher s'exerce par le contact des sensibles [tactiles] eux-mêmes; il perçoit par lui-même» 46 et non par l'intermédiaire d'un médium extérieur (à savoir un diaphane).

Mais la nature ontologique de la chair est semblable à celle du diaphane, c'est-à-dire qu'il s'agit également d'un composé intelligible dont la substance et la forme sont immatérielles. Comme le diaphane, la chair rend saisissable ce qui est intelligible dans le sensible, en l'occurrence le sensible tactile. Les sensibles immédiats sont, à leur tour, rendus proprioceptifs par l'acte de la chair: leur saisie est à la fois un constat et une compréhension.

La faculté du toucher, relevant de la chair, s'inscrit directement dans la survie biologique du corps animé en tant qu'instance qui garantit sa longévité et dont la privation entraîne la mort ${ }^{47}$. Tandis que la chair sollicite le corps en tant qu'unité biologique dont l'unique souci est le maintien temporel du Moi de l'organisme, le diaphane sollicite le corps en tant que véhicule mobile d'un Soi, dont l'unique souci est d'assurer un déploiement sensoriel spatial et de garantir la permanence des jouissances panoramiques.

À travers les médiums diaphane et chair, diverses formes du même genre peuvent être actualisées et virtualisées sur un même champ sensoriel, sans que l'identité essentielle de celui-ci soit mise en péril par cette modification, c'est-àdire sans que le potentiel de la sensation disparaisse. C'est le médium qui transfere une empreinte intelligible de l'objet perçu (source/agent) à l'organe de la faculté sensitive (cible/ patient) du percevant. Le principe d'organisation formelle qu'on a dégagé jusqu'à maintenant est sous-jacent, selon Aristote, aux cinq sens canoniques. Le visuel, comme l'auditif, l'olfactif, le gustatif et le tactile, se comportent selon le même processus d'altération formelle à travers des actants positionnels: source, médium, cible.

\section{Les actants positionnels}

\section{et les schèmes actantiels isomorphes}

Pour Aristote, la sensation est un état d'âme ayant son propre logos. Celui-ci s'obtient à travers une dynamique de fusion entre les actants positionnels source et cible et s'établit sous l'impulsion des médiums diaphane et chair, qui instaurent une intelligibilité sui generis présidant à la scission du sensoriel et de sa substance.

Sensation et intelligibilité ne font qu'un dans l'aisthêsis, car elles sont engendrées par un même acte constitutif: une «ceptivité» originelle, selon Aristote, «le premier mouvant», une entité intelligible qui, en acte, vient nourrir certains effets sensoriels qui deviennent, extéroceptivement, intéroceptivement et proprioceptivement, saisissables. En quelque sorte, nous pourrions considérer la "ceptivité" comme une énergie intelligible; une sorte de conceptualisation d'une prégnance indifférenciée. Tout ce qui est rendu saisissable par le médium se manifeste donc dès l'abord comme une forme investie d'une "énergie plus ou moins vive» ${ }^{48}$, qui rend la saisie de cette forme intense et, par conséquent, susceptible de produire un effet intelligible que l'on appelle iconicité: l'autonomisation morpho-sensorielle de la "ceptivité».

Ce processus de privation cause une altération dans l'organisme, qui se fait entendre soit comme une altération sui generis de l'expérience sensorielle en tant que telle, conforme à la nature sensitive de l'être animé, soit comme une altération perfective, conforme à la nature ontologique de l'être animé.

En ce qui concerne l'altération sui generis, les actants positionnels médium, source et cible mettent en place des modes d'existence dont la logique correspond à la logique de la sensation. L'actualisation d'une certaine couleur par l'intermédiaire du diaphane engendre un potentiel, à savoir un champ sensoriel spécifique: "c'est du blanc». Cette actualisation virtualise toutes les autres couleurs que ce champ sensoriel peut retenir. Mais le fait que le champ sensoriel puisse devenir éventuellement jaune dépend de la permanence de l'identité du champ sensoriel en tant qu'espace de qualités. La permanence du champ sensoriel potentialise des scénarios canoniques sensoriels à partir desquels une autre actualisation est toujours pensable. Soitle scénario suivant: actualisation $\rightarrow$ potentialisation $\rightarrow>$ virtua lisation $\rightarrow$ actualisation.

Dans ce premier cas, la logique de la sensation n'est pas une logique dont l'intelligibilité est purement mécanique. 
Il ne s'agit pas d'une finalisation des sensations à travers une programmation du sensoriel, mais d'une logique qui fait naître des domaines sensoriels où le percevant, en tant que centre déictique, symbolise spatialement avec le monde: "c'est du blanc; le blanc est ici; c'est blanc et c'est grand". Cette stabilisation esthétique montre dans quelle mesure la sensation a sa propre raison d'être, qui est celle, comme le dit Jean-François Bordron, d'une «logique du diagramme: il ne s'agit pas tant d'atteindre une fin que de constituer un domaine à partir d'un parcours libre» ${ }^{49}$.

La réalisation des formes sensibles en tant que formes sémiotiques n'intervient que lorsqu'il est question d'un maintien en présence d'au moins une forme sensible. Cette temporalisation du sensoriel a pour conséquence que l'opération axiologique de la sensation est suspendue en faveur d'un saut téléologique: «c'est blanc, c'est grand: c'est le fils de Diarès; c'est amer, c'est jaune: c'est du fiel", qui permet non seulement de stabiliser le flux sensoriel, mais également de le finaliser, c'est-à-dire de le stabiliser sémiotiquement. On a affaire ici à ce que J.-F. Bordron appelle une logique du programme, à travers laquelle l'action autonome du sensoriel trouve une détermination réalisée: "c'est du fiel». Le scénario est dès lors le suivant: actualisation $\rightarrow$ potentialisation $\rightarrow$ virtualisation $\rightarrow$ réalisation.

La différence entre une logique du diagramme et une logique du programme réside dans le fait que, dans la première, la sensation est une action non orientée, tandis que, dans la seconde, la sensation est soumise à une finalisation: une quête de sens ou de beauté ${ }^{50}$. Qu'il s'agisse d'une logique du programme ou d'une logique du diagramme, la sensation est pour Aristote toujours un acte, un devenir, voire une émulation entre la temporalité limitée de l'organisme du percevant et l'extension spatiale du monde. La sensation étant le résultat de cette émulation, elle enveloppe le percevant et le monde, tout comme la forme enveloppe la matière, c'est-à-dire en en donnant une définition autonome ${ }^{51}$.

Les analyses de la sensation, telles qu'Aristote les présente dans De Anima et «De Sensu», n'ont pas pour but de dégager la signification de la sensation comme un acte subjectif, mais, au contraire, de nous offrir, comme il le dit lui-même, "un exposé schématique» 52 . En objectivant la sensation comme un logos, Aristote semble viser une mise en réseau de toutes les modalités sensorielles. Bien que disposés dans des champs sensoriels différents, les cinq sens canoniques font appel à des structures isomorphes: un médium qui engendre un champ sensoriel en activant un agent-source et un patient-cible. L'établissement d'un réseau d'analogie - «la simultanéité de sensation relativement au même objet " 53 - ne revient pas à un seul sens, ni à un sixième sens, il est plutôt le travail des «sensibles communs» et la «sensation commune» dont il sera question un peu plus loin.

\section{Les sensibles communs}

Définitions:

Les sensibles communs sont le mouvement, le repos, le nombre, la figure, la grandeur; les sensibles de ce genre ne sont propres à aucun sens, mais sont communs à tous. ${ }^{54}$

Toutes ces déterminations, en effet, c'est par un mouvement que nous les percevons: ainsi, c'est par un mouvement que nous percevons la grandeur, et par suite, aussi la figure, car la figure est une certaine grandeur; la chose en repos, c'est par l'absence de mouvement; le nombre, c'est par la négation de la continuité et aussi par les sensibles propres, puisque chaque sensation n'a qu'un seul objet. ${ }^{55}$

Le fait que les sensibles communs se retrouvent aussi dans un autre sensible montre clairement que chacun d'eux est quelque chose de tout différent. ${ }^{56}$

Les sensibles communs sont des sensibles collectivement communs aux sens particuliers. Leur mise en jeu coïncide avec l'actualisation synergique d'au moins deux sens propres. Les sensibles communs sont perceptibles par soi et assurent la cohésion superficielle de la sensation en lui procurant un moment d'unité. Ils garantissent son maintien en présence en lui donnant un endroit fixe, voire un sens de volume. Mais puisqu'ils font partie de la sensation commune, c'està-dire de la sensibilité en général, ils assurent également, en tant que sensible par accident, la cohérence de la sensation en lui procurant une homogénéité qui va au-delà du simple constat d'existence sensorielle et qui permet un jugement épistémique de la sensation.

Aristote reconnaît cinq sensibles communs canoniques: le mouvement, le repos, le nombre, la figure et la grandeur, qui se ramènent au seul axe général de la spatio-temporalité ou du mobile, c'est-à-dire ce qui est en mouvement et se déplace d'un point jusqu'à un autre ${ }^{57}$. C'est pourquoi nous allons traiter les sensibles communs comme des sensibles 
sensori-moteurs affectant la sensibilité dans sa collectivité. Autrement dit, le sentiment de la sensori-motricité, à savoir le sens de déplacement ou d'immobilité, est naturellement conjoint à la sensation de tous les objets sensibles et attribue un moment cinétique objectif à une perception subjective.

Un exemple, venant de De Anima, illustre la façon dont les sensibles communs ajoutent une charge sensori-motrice ou cinétique à la reconstruction d'une présence sensible: "Le blanc est ici» 58 (sensible propre + sensible commun). En tant qu'accident de la sensibilité du sensible propre «blanc», le sensible commun «ici» (absence de mouvement; repos) déictise la sensation en lui assignant un endroit fixe. Le sensible propre "blanc» est accompagné par un geste déictique qui opère un maintien en présence temporel de ce qui est en mouvement, c'est-à-dire la sensation que l'on a du «blanc». La signification de «ici» n'est pas canoniquement liée aux sensibles propres, mais paradigmatiquement. En effet, la signification de "le blanc est ici» (ou de «le blanc est grand») relève d'une position spécifique de notre corps et dépend de l'activité sensori-motrice du percevant qui cherche à se situer vis-à-vis d'une présence qui surgit dans son champ sensoriel.

Les sensibles communs se manifestent en tant qu'avatars $\mathrm{du}$ «mobile» dès qu'on a la sensation d'un sensible propre. C'est pourquoi Aristote les considère comme des sentis par soi ${ }^{59}$. On sent essentiellement, conformément à la nature de la sensibilité, le mobile en tant que mobile au même titre que le blanc en tant que blanc ${ }^{60}$. Ils ne sont pas saisis par l'intermédiaire des sensibles propres, mais plutôt dans les sensibles propres et les formes que ceux-ci retiennent de la substance-matière du sensible.

D'où l'ambiguïté qui entoure le statut des sensibles communs. D'un côté, ils sont des accidents des sensibles propres vis-à-vis desquels ils sont ontologiquement disjoints: «nous les percevons par accident au moyen de chaque sens ${ }^{61}$. Il ne s'agit pas, dans l'acte de la sensation, d'une concomitance ou d'une simultanéité par nécessité entre le sensible propre et le sensible commun, mais d'une conjonction par possibilité distributive. Le statut des sensibles communs est en l'occurrence paradigmatique: l'actualisation d'un seul sensible commun par un sensible propre et, à l'inverse, d'un sensible propre par un sensible commun, virtualise dans le même temps les autres sensibles communs et, a fortiori, les autres sensibles propres, que l'on peut saisir, les uns comme les autres, par accident, dans la mesure où il sont disponibles au moment de l'actualisation d'un seul sensible. D'un autre côté, ils ne sont pas des accidents par rapport à la sensibilité en général, car ils s'incarnent comme une sensation commune directement dans la sensation du sensible dont ils sont dérivés.

Il s'ensuit que l'on ne saurait leur attribuer aucune faculté sensitive qui leur soit spécifiquement afférente. En effet, la grandeur, que perçoit la vue, est perçue par et dans la couleur. Mais cette grandeur peut être perçue également par le toucher dans un tangible. Si l'on accepte ce point de vue, leur statut en tant que sensible est, dès lors, un statut par soi et non par accident.

\section{Modulation}

Eu égard à la différence, soulignée par Aristote à maintes reprises, entre sensibles à distance et sensibles immédiats, les sensibles communs assurent un maintien en présence $\mathrm{du}$ flux sensoriel dans la mesure où ils procurent aux sensibles extéroceptifs qui spatialisent l'expérience sensorielle (la vue, l'ouïe, les parfums) un maintien temporel et aux sensibles proprioceptifs qui temporalisent l'expérience sensorielle (le toucher, le goût, les aromates) un maintien spatial. Dès lors que les sensibles temporalisants peuvent être des accidents des sensibles spatialisants ("c'est blanc, c'est doux " ${ }^{62}$ ) et, à l'inverse, dès lors que les sensibles spatialisants peuvent être, à leur tour, des accidents des sensibles temporalisants (“c'est amer, c'est jaune» ${ }^{63}$ ), les sensibles communs garantissent leur communication intermodale de façon à permettre de prononcer que deux qualités ne forment qu'un seul objet sensoriel: "c'est amer, c'est jaune [et d'une certaine grandeur]: c'est du fiel» ${ }^{64}$. Dans l'un comme dans l'autre cas, le maintien est accompagné d'une charge cinétique relevant de la présence corporelle du percevant comme centre de gravité et de repérage pour tous les mouvements.

Les sensibles communs relèvent d'une activation générale et pondérée des réseaux sensoriels. Grâce à cette activation pondérée, à partir d'un seul type de stimulation tous les types sensoriels sont affectés. Les sensibles communs fonctionnent, alors, comme des modulations cognitives dont l'organisation iconique sémiotique est le corrélat. En tant que tels, ils apparaissent comme des schèmes émergents synchronisant le flux sensoriel et l'activité sensori-motrice et perceptive du percevant. 
Les sensibles «par accident»

Définitions:

On dit qu'il y a sensible par accident si, par exemple, on perçoit le blanc comme étant le fils de Diarès. C'est par accident, en effet, que l'on perçoit ce dernier, parce qu'au blanc est accidentellement uni l'objet senti. ${ }^{65}$

La sensation des sensibles propres est toujours vraie, ou, du moins, sujette le moins possible à l'erreur. La perception que ces sensibles propres sont des accidents vient en second lieu. ${ }^{66}$

Ce qu'Aristote appelle un sensible par accident $\mathrm{a}$ trait à ce qui se trouve accidentellement uni à un objet sensible extéroceptif et qui s'actualise intéroceptivement. Une couleur qui évoque un certain objet, par exemple, ou le sens de la vue, qui évoque le doux, un sensible propre au tactile. Le cas échéant, le sensible propre, en l'occurrence le tactile, s'obtient comme «accident» de la vue. Le sensible par accident se produit quand il y a simultanéité de sensations relativement au même objet, mais il n'est jamais directement subi par la sensation. Un «accident sensoriel» affecte la sensation seulement indirectement par les sensibles propres et les sensibles communs, qui leur sont conjoints. Il est impossible de sentir directement le "fils de Diarès", cher à Aristote, dont on ne subit pas l'empreinte, tandis qu'on sent directement le «blanc» de son manteau.

La saisie d'un sensible par soi se fait toujours sans erreurs, mais sa visée - les réactions psycho-physiologiques intéroceptives qu'il suscite (ses accidents) - est sujette, selon Aristote, à l'illusion. Lorsqu'on passe d'une expérience sensorielle à sa finalisation à travers les possibles inférences, il convient donc de parler d'une gradation entre une perception d'un sensible par soi, qui est épistémologiquement objective et phénoménologiquement forte, et la perception d'un sensible par accident, qui est épistémologiquement subjective et phénoménologiquement faible.

\section{Modulation}

Tandis que le fonctionnement des sensibles propres relève d'un processus neuro-sensoriel de calcul ou de mélange et celui des sensibles communs d'un processus de synchronisation, le sensible par accident, lui, relève d'un processus de convergence en ce que le sensoriel est finalisé autour d'un seul sensible propre en établissant un faisceau: «c'est blanc, c'est grand: c'est le fils de Diarès».

\section{La sensation commune}

Pour Aristote, la sensation est un phénomène strictement physiologique, et l'explication qu'il a mise en place vise essentiellement à mieux comprendre l'unité du système sensoriel et ses possibles scénarios. Cette unité dépend d'un mécanisme sensoriel, la koinè aisthêsis, qui a trait à la communauté formée par l'ensemble des sensibles.

Dans De Anima, Aristote rapporte à ce mécanisme trois caractéristiques, qui ne peuvent être attribuées à un sensible propre en particulier:

1. la saisie des sensibles communs; 2 . la réflexivité; 3 . la synthèse sensorielle: la mise en relation et la différenciation de toutes les informations sensorielles dans la saisie d'un même objet.

\section{Première définition: saisie des sensibles communs}

Il n'est pas possible non plus qu'il existe un organe sensoriel spécial pour les sensibles communs, que nous percevrions ainsi par accident au moyen de chaque sens. ${ }^{67}$

En réalité, des sensibles communs nous avons déjà une sensation commune. 68

Comme nous avons amplement examiné les sensibles communs, nous nous contenterons de constater que la sensation commune élargit la sensation des sensibles propres en les ouvrant mécaniquement à d'autres sensibles par soi, en l'occurrence aux sensibles communs: «c'est blanc, c'est grand». En donnant une certaine étendue et, par conséquent, une charge sensori-motrice à la saisie de la sensation, les sensibles communs ouvrent la sensation à une véritable logique cognitive. Notons également que les sensibles communs sont communs à la sensation au sens paradigmatique, tandis que la sensation commune est commune à la sensation au sens ontologique, c'est-à-dire associée à chaque incidence sensorielle séparément.

\section{Deuxième définition: la réflexivité}

Puisque nous percevons que nous voyons et entendons, c'est nécessairement ou bien par la vue que le sentant perçoit qu'elle voit, ou bien par un autre sens. ${ }^{69}$

Posons [...] que chaque sens en particulier possède, d'une part, quelque chose de propre, d'autre part, quelque chose de commun; ainsi la vue possède en propre le voir et l'oüe l'entendre, mais il y a aussi une faculté commune qui les accompagne tous. 70 
La sensation commune opère une identification du sensible propre par calcul intramodal: «la vue juge du blanc et du noir, le goût, du doux et de l'amer. Et il en est de même aussi pour les autres sens» ${ }^{71}$. La faculté de juger, inhérente à la sensation d'un sensible propre, est basée sur l'affect que cause la sensation. La sensation se sent ellemême, parce qu'elle est quelque chose de nous-mêmes. Elle est un progrès, un devenir affectif de notre nature sensible. Selon cette deuxième définition, la sensation - l'action qu'exerce le sensible sur le sens - n'est pas mécanique, mais téléologique. Le sensible est une fin vers laquelle se porte le sens qui actualise le sensible en même temps.

Aristote compare dans De Anima la sensation commune avec le point géométrique, qui, lui, est indivisible en tant qu'unité, mais divisible en tant que segment d'une ligne ${ }^{72}$. La sensation commune juge les segments - les objets sensibles - sur la "ligne dynamique» de la sensation de deux façons différentes: comme deux qualités à la fois divisées et, par conséquent, unies par la sensation commune, qui fonctionne comme une limite jugeante ${ }^{73}$. Dans un acte instantané de saisie, elle juge que le gris n'est pas blanc et que le blanc n'est pas noir. La sensation commune est alors une charnière, une articulation entre deux sensibles contraires: «c'est donc en un même temps que la faculté [la sensation commune] prononce: elle est par suite une inséparable unité en un temps inséparable» ${ }^{74}$.

\section{Troisième définition du sens commun:}

\section{synthèse sensorielle}

C'estpar accident que les divers sens perçoivent les sensibles propres les uns des autres; ils agissent alors non pas en tant que sens séparés, mais en tant que formant un seul sens, quand il se produit simultanéité de sensation relativement au même objet; c'est le cas, lorsque nous percevons que le fiel est amer et jaune: car iln'appartient certainement pas à un autre sens de prononcer que ces deux qualités ne font qu'une seule chose. 75

Quant au principe [c'est-à-dire le sens commun] par lequel l'âme juge que le doux differe du chand [...]: ce principe est une chose une, et une au sens où la limite est une. Et ces sensibles, le sens commun, qui est un par analogie et par le nombre, les possède en lui dans le même rapport l'un à l'égard de l'autre que ceux-ci se trouvent, en réalité, vis-à-vis l'un de l'autre. ${ }^{76}$

La troisième fonction de la sensation commune est de procurer à la sensation la capacité d'établir des synthèses en opérant une unification intermodale entre les sensibles propres de divers genres. La synthèse s'obtient en temps essentiel, c'est-à-dire synchroniquement dans le mouvement de l'acte, pendant que les sensibles sont sensibles simultanément et non en temps accidentel, c'est-à-dire dans l'après-coup de l'affirmation ${ }^{77}$.

La sensation commune a, dès lors, une «conscience». Il ne s'agit pas de la conscience d'un «Soi» identique à travers le temps et constant au cours des épisodes variés de la vie psychique, ni même de la conscience d'un «Soi» instantané, synthétisant dans l'unité d'une expérience une diversité d'événements perceptifs, affectifs, cognitifs, mais seulement de la "conscience" ponctuelle propre à chaque épisode perceptif ${ }^{78}$. Par l'intermédiaire de cette troisième fonction, l'ensemble des sensibles propres est intégré, c'est-à-dire investi d'un affect qui finalise la saisie afin de créer une unité synesthétique: «c'est du fiel».

\section{Modulation}

La sensation commune n'est nullement un sixième sens ou une sorte de "homoncule», mais un sens qui fonctionne comme un principe de connectivité faisant le lien entre les divers sensibles propres par soi et par accident. Elle nous procure la possibilité de juger de la différence entre, par exemple, doux et chaud. Elle assure la congruence de l'univers sensoriel en lui procurant un effet global d'une totalité polysensorielle. Mais, dans le même temps, elle garantit la cohérence au niveau superficiel de la sensation en ce qu'elle réalise la discrimination pondérée des sensibles propres. La sensation commune a pour fonction d'englober, d'unifier les sens afin de créer un réseau, c'est-à-dire, comme le dit Barbara Cassin, "de mettre en rapport ces rapports littéralement idiots que sont les sentis et la sensation, donc de juger de leur différence et de la dire» 79 . À travers la sensation commune, les sensibles à distance et les sensibles immédiats s'intériorisent et constituent un champ sensoriel intériorisé où la sensation («c'est jaune, c'est amer», «c'est blanc») est convertie dans des objets iconiques transversaux ("c'est du fiel; c'est le fils de Diarès"), qui ne sont nullement les objets sensibles tels que nous les retenons à travers les sensibles propres, mais les objets intéroceptifs susceptibles de devenir «le sujet habituel de nos phrases, ou le sujet de nos phrases habituelles» ${ }^{80}$.

Le type de connexion propre à l'activité neurosensorielle de la sensation commune est celui de la tranversalisation. 
Les sensibles propres relevant de sens différents sont rassemblés sur l'unité d'un même objet.

\section{Synthèse sémiotique:}

\section{comment la signification vient-elle à la sensation? ${ }^{81}$}

Nous avons vu que le principe de l'aisthêsis part de ce qui se fait sentir immédiatement, sans erreurs de jugement, et qui représente par là même l'essence du sensible, c'est-àdire, pour Aristote, la forme qu'on retient de la substance. Cette expérience esthétique immédiate est celle des sensibles propres qui, grâce à la médiation des sensibles communs, sont susceptibles de former un "quelque chose" qui se tient et qui nous procure un "constat d'existence». La réaction réflexe aux stimuli sensoriels se trouve momentanément suspendue et procure un effet de présence. On est effectivement en présence, comme le dit Jean-François Bordron, d'un moment d'iconisation, comme le maintien spatiotemporel d'une configuration sensorielle provisoirement stabilisée ${ }^{82}$. Cette stabilisation du flux sensoriel s'obtient lorsque le mouvement des stimuli sensoriels est interrompu par la force immobilisatrice des sensibles communs et que le percevant s'arrête pour devenir «autre»; c'est-à-dire lorsque s'opère une dissociation de la forme et de la substance, qui est à la fois ressentie et opérée par le percevant, et qui préfigure la scission ontologique entre l'instance de discours et le discours-énoncé autonome dans lequel celuici se manifeste. Le sensoriel devient d'abord un scénario non finalisé, qui affecte le percevant avant de devenir un moment iconique dans un discours qui porte sur le sensoriel. Cette première dissociation représente un débrayage sensoriel constitutif, dans la mesure où le percevant se rend compte que son devenir «autre» a affaire aux formes sensorielles qu'il retient du flux sensoriel. Pour que la sensation devienne effectivement apte à être rapportée à un discours, il faut que l'iconisation, l'établissement de simples rapports sensoriels entre les sensibles propres, trouve son moment d'unité synesthétique. Cette conversion s'opère par l'intermédiaire de la sensation commune et a pour résultat l'établissement d'un icône synesthésique.

Le point de départ de l'analyse que nous offre Aristote de la logique de la sensation réside dans le calcul constitutif d'un sensible propre par soi et l'incontestable constat d'existence sensorielle que celui-là nous procure. Éprouver une expérience sensorielle veut dire qu'un percevant, en tant que siège corporel d'une orientation sensible, opère un calcul sur ce qu'il ressent en filtrant et stabilisant l'énergie du flux sensoriel. Par là même, il ouvre une perspective égocentrique susceptible de procurer un sentiment de cohésion au monde sensible. Mais cette prise de position implique que l'expérience sensorielle non seulement affecte le percevant qualitativement, mais également quantitativement en ce qu'elle impose sa propre position, orientation et étendue. En effet, le percevant ressent que "c'est blanc» et constate que "ce blanc est ici, grand, mobile».

À partir de la première identification d'une présence sensible, s'établissent ainsi deux dimensions perceptives:

1. Une dimension relative à l'affect et à l'imagination que déclenche la sensation d'une seule qualité sensorielle auprès d'un percevant qui cherche à "opiner au sujet de la chose même qu['il] sent ${ }^{83}$, c'est-à-dire qui cherche à rendre intelligible ce qu'il ressent en focalisant son attention sur l'énergie émanant d'une seule qualité sensorielle pour en inférer, à travers un mouvement secondaire, les accidents successifs ${ }^{84}$ : "ce blanc, qui est grand, est fils de Diarès" (sensible propre par soi $\rightarrow>$ sensible commun par accident ${ }^{85}$ $\rightarrow$ sensible par accident). Le constat qu'on a affaire au «fils de Diarès» s'obtient à travers un processus d'iconisation téléologique relevant d'un affaiblissement phénoménologique progressif en faveur d'une augmentation de l'intensité affective et imaginaire. La canalisation intéroceptive de cette intensité en un axe de profondeur perceptive à partir d'un centre d'orientation est alors tributaire de l'énergie issue du «blanc».

2. Une dimension relative au constat que chaque présence sensible occupe une position spatio-temporelle. Chaque présence sensible est accompagnée de propriétés externes d'une certaine étendue qui sont critiques pour sa saisie et qui contrôlent la sensation sensori-motrice du percevant. La conversion de la sensori-motricité en un axe de profondeur perceptive relève de la position égocentrique spatiotemporelle du percevant relative à la position du stimulus sensible: «le blanc est ici/immobile» (sensible propre par soi $\rightarrow$ sensible commun par soi). En l'occurrence, le «blanc» se trouve à un endroit fixe par rapport à l'endroit où se trouve le percevant, qui est susceptible de saisir et de subir le stimulus en tant que quantité issue de ce «blanc». Au lieu d'être finalisé, le sensoriel n'est maintenu en présence que comme un événement qui affecte la position du percevant. On a affaire à deux axes qui se mettent en place depuis une seule présence égocentrique: 
1. Un axe (valence intensive) d'intensité ou de visée, que nous avons identifié à un mouvement téléologique dont l'orientation est celle d'une finalisation iconique à partir d'un constat d'existence sensorielle épistémologiquement neutre. 2. Un axe (valence extensive) de déploiement spatio-temporel, que nous avons identifié à un mouvement sensorimoteur dont l'orientation est celle d'une hétérogénéisation à partir d'un constat d'existence homogène (un simple mélange).

Corrélés l'un à l'autre, ces deux axes sous-tendent quatre grands types de connexion: le mélange, la synchronisation, la convergence et la transversalisation, qui, à leur tour, représentent les quatre modulations sous-jacentes à quatre zones tensives typiques, susceptibles d'accueillir et de caractériser différents types de valeurs sensorielles: le sensible propre, le sensible commun, le sensible par accident et la sensation commune, dont les modes sémiotiques sont la discrétisation, l'intégration, la concentration et l'unification.

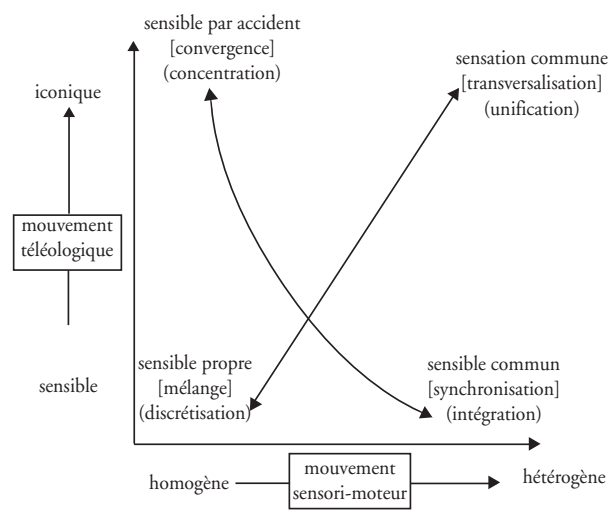

\section{Conclusion}

Connaître la sensation, c'est, pour Aristote, la connaître par ses principes constitutifs. La démarche aristotélicienne est avant tout une analyse qui consiste à mettre en évidence ces principes en récusant d'avance toute interprétation empiriste: l'immédiatement connu est une généralité mélangée, qui n'est qu'un point de départ susceptible de nous amener aux particularités distinguées et intelligibles.

Nous avons vu que la description du sensoriel se fait à partir des sensibles propres, mais au quotidien nous n'avons jamais d'emblée une expression séparée de ceux-ci. Car si la sensation nous était livrée par ses seuls éléments constitutifs, ces éléments seraient épistémologiquement neutres, c'est-à-dire que leur mélange n'appartiendrait pas unilatéralement à la sensation. Or, nous ne connaissons rien d'autre que la relation spécifique qui les coordonne pour en faire une sensation polysensorielle. Les sensibles propres peuvent, de ce fait, entrer dans une combinaison intermodale sans toutefois changer épistémologiquement de statut. Aristote maintient leur monodimensionnalité: le fiel n'est jamais senti en tant que fiel, mais toujours comme jaune, amer, en acte.

\section{NOTES}

1. Dans la terminologie de Peirce, il est question de trois sortes de signes iconiques, à savoir le qualisigne, le sinsigne et le légisigne. $C f$. N. EveraertDesmedt, 1990 :53-61; voir sur Internet: http://perso.wanadoo.frlala/Peircel pragmatisme.htm.

2. Pour une sémiotique d'inspiration peircienne, voir P.Å. Brandt, 1994 et 1999-2000: 81-87.

3. J. Courtés, 1992: 85.

4. C. Metz, 1972: 161-162.

5. U. Eco, 1970: 14 .

6. Ibid., p. 21.

7. U. Eco, 1998: 15-16.

8. A.J. Greimas, 1983: 46 .

9. A.J. Greimas, $1984: 9$.

10. J.-M. Floch, 1982: 203.

11. J.-M. Floch, 1986a: 120.

12. J.-M. Floch, 1986b: 169.

13. A.J. Greimas et J. Fontanille, 1991.

14. J. Fontanille et C. Zilberberg, 1998; J. Fontanille, 1999a.

15. J. Fontanille, 1999b: 10.

16. J.-L. Poirier, «Introduction», dans Aristote, 1990: 7-8.

17. Aristote, De l'âme (De Anima, 1995, désormais DA), II, 2, 424a 17-24, p. 139-140. 
18. Nous suivons ici les propos de H. Parret, paraphrasant Aristote, DA, III, 1, 425 b3-5 (H. Parret, 1993: 63).

19. J.-P. Dumont, 1992: 102.

20. Aristote, «Mouvement des animaux », 4,700 a 8, cité par J.-P. Dumont, 1992: 106

21. DA, III, 2, 425 b 26.

22. B. Cassin, 1997: 119.

23. DA, II, 6, 418 a 9.

24. $D A$, II, 6,418 a 25 .

25. DA, II, 6, 418 a 21.

26. $D A$, III, 1,425 b 1 .

27. Cette première segmentation est fidèle à la présentation que nous offre Aristote lui-même dans DA, II, 6-12; III, 1-2.

28. DA, II, 5, 417 b 1-18; II, 5, 418 a 5, «Dans l'acte, pâtir et agir sont identiques». Voir Aristote, 1990 : livre III, chap. 2, 202 b.

29. "Les états [sensoriels] ne sont pas des corps, mais une certaine affection et un certain mouvement sans quoi ce phénomène [la sensation] ne se produirait pas. Toutefois, ils ne se produisent pas non plus sans un corps". Aristote, «De Sensu (Des Sens)», dans Aristote, 2000: 6, 446 b 25.

30. DA, II, 2, 414 a 11-14.

31. Aristote, «De Somno (Du Sommeil)», dans Aristote, 2000: 1, 454 a 9.

32. DA, II, 1, 412 b 19. «Si l'œil était un animal complet, la vue en serait l'âme».

33. Il en va de même pour les autres sensibles propres par soi: «le raisonnement est encore le même pour le son et l'odeur; pour le toucher et le goût, il en est de même, en dépit des apparences». DA, II, 7, 419 a 25, 30. 34. La traduction proposée par Tricot est "mixtion».

35. Aristote, 1950: I, 10, 328 b 25.

36. Dans les textes d'Aristote, le statut d'odorat est particulier. Bien que l'odorat s'exerce au moyen d'un médium étranger au corps (l'air ou même l'eau, c'est-à-dire l'air humide), il dépend, au moins pour une partie de sa sémiotisation, du goût, dans la mesure où «les odeurs ont pris leurs noms [...] [des saveurs] en vertu de la ressemblance des choses : l'odeur douce, en effet, vient du safran et du miel, et l'odeur aigre, du thym et de choses de ce genre» ( $D A$, II, 9, $421 \mathrm{~b}$ 3). Il s'ensuit qu'Aristote prend en considération l'existence de deux sortes d'odeurs: d'une part les aromates «les odeurs qui correspondent aux saveurs [...] et qui contiennent par accident l'agréable et le désagréable» («De Sensu », 2000, 5, 443 b 20) : «les odeurs aigres, douces, âpres, astringentes et grasses» (ibid., 5, 443 b 4, 10) et, d'autre part, les odeurs "agréables en soi, comme par exemple celles des fleurs" (ibid., 443 b 27).

37. DA, II, 11, 423 b 13-17. C'est nous qui soulignons pour mettre l'accent sur l'aspect temporel du toucher.

38. $D A$, II, 7, 418 b 7 .

39. Observons également que, vu l'exigence logique que la faculté sensitive est en puissance, telle que le sensible est déjà en acte, les deux médiums diaphane et chair - ne peuvent actualiser la faculté sensitive et, par là même, engendrer un champ sensoriel qu'à la condition que l'organe sensoriel (la facultéen puissance) soit constituélui-même d'une qualité susceptible d'être activée par le médium. C’est ainsi que, selon Aristote, «la pupille est formée d'eau, l'ouïe d'air et l'odorat de l'un ou de l'autre" (DA, III, 1, 425 a 5) et que l'organe du toucher «n'est formé ni de terre, ni d'aucun autre élément pris isolément, mais que c'est un mixte de terre et de ces éléments [air et eau]" (DA, III, 13, 435 b 3; DA, II, 423 a 14). Diaphane et chair sont, dès lors, présents en puissance dans le corps animé.

40. DA, II, 7, 418 b 9 .

41. Rappelons que pour Aristote l'intellection est analogue à la sensation, toutes deux étant des facultates judicandi (DA, III, 4, 429 a 13). Voir aussi le commentaire de Tricot (ibid., p. 173).

42. $D A$, II, 11,423 a 6 .

43. $D A$, II, 10,422 a $7-8 ; D A$, II, 11,423 a 15-17.
44. $D A$, II, 11, 423 b 19.

45. DA, II, 11, 422 b 33 .

46. $D A$, III, 13,435 a $17-19$.

47. DA, III, 13, 435 b 4 .

48. Terminologie de la sémiotique tensive. Voir à ce sujet J. Fontanille, 1998: 64-72.

49. J.-F. Bordron, 1998: 98-99.

50. Comme le constate également J. Fontanille en paraphrasant Bordron: «la logique du programme est une logique d'enchaînement dirigée par un objectif, alors que celle du diagramme n'est dirigée que par le souci d'une différenciation progressive». J. Fontanille, 1999a: 24.

51. Cf. Aristote, 1990 : livre III, chap. 11, 207 a 35-207 b 1: «L'infini est contenu lui aussi : tout comme la matière $[. .$.$] ; c'est la forme qui contient ».$

52. DA, II, 11,424 a 15.

53. DA, III, 1,425 a $32-425$ b 1 .

54. $D A$, II, 6,418 a $17-19$.

55. DA, III, 1,425 a 16-19.

56. DA, III, 1, 425 b $4-9$.

57. DA, III, 2, 425 a 16-20; «De Sensu», 6, 446 a 29; 7, 449 a 20-21.

58. $D A$, II, 6,418 a 16 .

59. DA, II, 6, 418 a 10-11.

60. B. Cassin, 1997: 59.

61. DA, III, 1,425 a $14-15$.

62. DA, III, 1,425 a 23.

63. $D A$, III, 1,425 b 1 .

64. DA, III, 1, 425 b 2.

65. DA, II, 6, 418 a 20.

66. $D A$, III, 3, 428 b 17-18.

67. $D A$, III, 1,425 a13.

68. DA, III, 1,425 a 27.

69. DA, III, 2, 425 b 11 .

70. «De Somno (Du Sommeil)», 2, 455, 12-17, dans Aristote, 2000.

71. DA, III, 2, 426 b 11-12.

72. DA, III, 2, 427 a 10-11. Voir également $D A$, III, 7, 431 a 21-30.

73. DA, III, 2, 427 a 14 .

74. DA, III, 2, 426 b 29.

75. DA, III, 1,425 a 30.

76. $D A$, III, 7,431 a $21-30$

77. DA, III, 2, 426 b 29.

78. J. Brunschwig, 1991: 466.

79. B. Cassin, 1997: 121.

80. Ibid., p. 127.

81. Cette synthèse sémiotique prend appui sur les propositions de la sémiotique tensive, telles qu'elles sont formulées notamment dans J. Fontanille, 1998.

82. J.-F. Bordron: propos recueillis au Séminaire intersémiotique, Paris, EHESS-CNRS-IUF, saison 1998-1999.

83. $D A$, III, 3,428 b 1

84. DA, III, 3, 428 b 10-12: «Puisqu' une chose mue peut en mouvoir une autre à son tour; que l'imagination est, semble-t-il, une sorte de mouvement et ne peut se produire sans la sensation, mais seulement dans les êtres sentants et pour des choses qui sont objets de sensation; qu'en outre, un mouvement peut être produit par la sensation en acte et que ce mouvement est nécessairement semblable à la sensation». Ce qui fait dire à J. Tricot: «le mouvement secondaire de l'imagination doit ressembler au mouvement primaire de la sensation" (Aristote, De l'âme, p. 171).

85. Le sensible commun "grandeur" est à considérer ici comme un sensible par accident dans la mesure où il est ontologiquement indépendant du sensible propre par soi «blanc». Il revient à «fils de Diarès" par conjonction auquel il procure, par conséquent, une charge sensori-motrice. Voir $D A$, III, 3,428 b $23-24$ 


\section{RÉFÉRENCES BIBLIOGRAPHIQUES}

ARISTOTE [1934] : De l'âme [De Anima], trad. de J.Tricot, rééd.1995, Paris, Vrin, coll. "Bibliothèque des textes philosophiques";

Vrin;

— [1990]: Leçons de Physique, trad. de J.-L. Poirier, Paris, Pocket, coll. "Agora les classiques";

[2000]: Petits traités d'histoire naturelle [Parva Naruralia], trad. de J.M. Morel, Paris, Flammarion.

BORDRON, J.-F. [1998]: "Réflexions sur la genèse esthétique du sens", Protée, vol. 26, no2, 97-104.

BRANDT, P.Å. [1994]: Dynamiques du sens, suppl.2, Aarhus, Aarhus University Press, coll. "Poetica et Analytica»;

— [1999-2000]: "Grounding Iconicity»", Visio, vol. 4, n 3, automne/ hiver.

BRUNSCHWIG, J. [1991]: «Les multiples chemins aristotéliciens de la sensation commune", Revue de métaphysique et de morale, vol. 96, n 4, 455474.

CASsin, B. [1997] : Aristote et le logos, contes de la phénoménologie ordinaire, Paris, P.U.F.

COURTÉS, J. [1992] : «Du signifié au signifiant : étude d'une bande dessinée de B. Rabier", Nouveaux Actes Sémiotiques, n²1-22, Limoges, PULIM,

$1-88$.

DUMONT, J.-P. [1992] : Introduction à la méthode d'Aristote, Paris, Vrin, coll. "Bibliothèque d'histoire de la philosophie".

ECO, U. [1970]: "Sémiologie des messages visuels», Communications, $\mathrm{n}^{\circ} 15$, Paris, Seuil, 11-51;

— [1998] : "Réflexions à propos du débat sur l'iconisme (1968-1998) ", Visio, vol. 3, no $1,9-31$.
EVERAERT-DESMEDT, N. [1990]: Le Processus interprétatif, introduction à la sémiotique de Ch.S. Peirce, Liège, Pierre Mardaga, coll. «Philosophie et Langage».

FLOCH, J.-M. [1982] : "Les langages planaires», dans J.-C. Coquet (sous la dir. de), Sémiotique. L'École de Paris, Paris, Hachette, 199-207;

_ [1986a]: Les Formes de l'empreinte, Périgeux, Pierre Fanlac; [1986b] : "Sémiotique plastique", dans A.J. Greimas et J. Courtés, 1986.

FOnTANille, J. [1998] : Sémiotique du discours, Limoges, PULIM;

[1999a]: "Modes du sensible et syntaxe figurative", Nouveaux Actes Sémiotiques, no61-62-63, Limoges, PULIM, 1-68;

— [1999b]: «Le corps de l'actant, propositions pour une schématisation narrative", Degrés, n 100, Bruxelles, ASBL Degrés, 1-22.

FontANille, J. et C. ZILBERBERG [1998] : Tension et Signification, Sprimont, Pierre Mardaga, coll. "Philosophie et langage».

GreIMAS, A. J. [1983] : Du Sens II. Essais sémiotiques, Paris, Seuil;

- [1984]: "Sémiotique figurative et sémiotique plastique», Actes Sémiotiques - Documents IV (60), Paris, INLF, 5-24.

GREIMAS, A. J., ET J. COURTÉS [1986] : Sémiotique. Dictionnaire raisonné de la théorie du langage, t. 2, Paris, Hachette, coll. "Langue, Linguistique, Communication".

GreimaS, A. J. et J. FONTANILlE [1991] : Sémiotique des passions, des états de choses aux états d'âme, Paris, Seuil.

METZ, C. [1972] : «Au-delà l'analogie, l'image », Essais sur la signification du cinéma, t. II, Paris, Klincksieck, 151-162.

PARret, H. [1993] : «(Syn)esthésies du visible», Versus, no 65-66, Bologne, Bompiani, 59-70. 